\title{
Model for the vaporization of mixed organometallic compounds in the metalorganic chemical vapor deposition of high temperature superconducting films
}

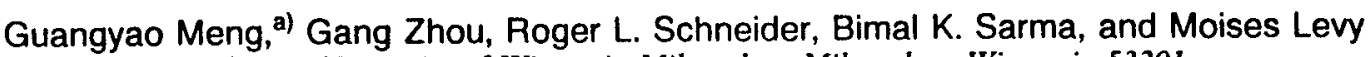 \\ Department of Physics, University of Wisconsin-Milwaukee, Milwaukee, Wisconsin 53201
}

(Received 4 March 1993; accepted for publication 25 July 1993)

\begin{abstract}
A model of the vaporization and mass transport of mixed organometallics from a single source for thin film metalorganic chemical vapor deposition is presented. A stoichiometric gas phase can be obtained from a mixture of the organometallics in the desired mole ratios, in spite of differences in the volatilities of the individual compounds. Proper film composition and growth rates are obtained by controlling the velocity of a carriage containing the organometallics through the heating zone of a vaporizer.
\end{abstract}

Metalorganic chemical vapor deposition (MOCVD) systems have produced excellent high- $T_{c}$ thin films. ${ }^{1}$ In the conventional multisource MOCVD system, it is difficult to control the gas phase composition and hence the film stoichiometry. This is because the volatility and stability of the organometallic compounds, which are individually vaporized, then mixed, strongly depend on temperature, system pressure, and carrier gas flow rate. Hiskes et $a l^{2}$ developed a single source MOCVD technique in which a specially designed vaporizer was employed. This vaporizer can be used to volatilize a stoichiometric mixture of $\beta$-diketonates of yttrium, barium, and copper to produce a mixed vapor in a 1:2:3 ratio, respectively, of the organometallics. This is accomplished even though the three compounds have significantly different volatilities.

In this letter, we offer a model which provides insight into the process of vaporizing mixed organometallic compounds to produce high quality thin films of $\mathrm{YBa}_{2} \mathrm{Cu}_{3} \mathrm{O}_{7}$ (YBCO). The model clearly shows that under steady-state conditions, the mixed organometallic vapor must have a stoichiometric ratio of the individual organometallics identical to that in the solid mixture.

A simplified schematic diagram of the vaporizer, which is part of our MOCVD system, is shown in 'Fig. 1. The organometallic compounds are finely ground and mixed well, and then packed into a thin walled, slotted glass tube. This slotted tube slowly traverses an abrupt temperature gradient between the cooling jacket and the heating lamp, which heats the mixed organometallics from room temperature to above $300^{\circ} \mathrm{C}$, which is above the highest vaporization temperature of the three $\beta$-diketonates. ${ }^{3}$ Steady-state vaporization of the powdered mixture occurs within the sharp temperature gradient and the vapors are swept from the vaporizer and transported to the substrate by a carrier gas which flows in the same direction as the slotted tube. This technique establishes a stream of mixed organometallic vapors with the desired composition.

In our model, for simplicity, we use an open channel

\footnotetext{
a) On leave from Department of Materials Science and Engineering, University of Science and Technology of China, Hefei 230026, People's Republic of China.
}

carriage as a container for the mixed organometallics, rather than the circular cross-sectional slotted tube, actually used in our vaporizer. The rectangular cross-sectioned carriage has an internal width, $w$, and height $h$, as shown in Fig. 1.

We assume (1) the mixed powder contains three organometallic compounds $A, B$, and $C$ in the volume concentrations $q_{A}, q_{B}$, and $q_{C}$ and that $q_{A}<q_{B}<q_{C} ;$ (2) all of the mixtures in any given layer are at the same temperature, and the surface area of the layer is constant until vaporization commences; and (3) the surface vaporization rates of the individual organometallic compounds, $r_{A}, r_{B}$, and $r_{C}$ are only a function of temperature and are not interdependent.

With $r_{B}<r_{A}<r_{C}$, for example, a specific composition profile of the powder will be established under steady-state conditions within the heating zone, as is shown in Fig. 2. The total vaporization rates of compounds $A, B$, and $C$ in units of, e.g. mol/s, are given by

$$
R_{i}=\int r_{i} w d l=r_{i} w l_{i}=r_{i} w \frac{h v q_{i}}{r_{i}}=w h v q_{i},
$$

where $l_{i}$ is a length along the total length of the melt, within the heating zone, in which component $i$ is present, and $v$ is the velocity of the carriage containing the mixed

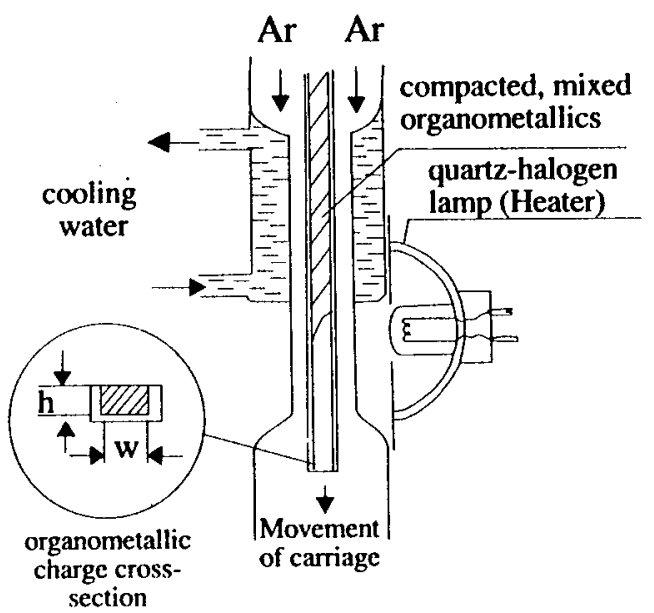

FIG. 1. The mixed organometallic compounds vaporizer. 
(a)

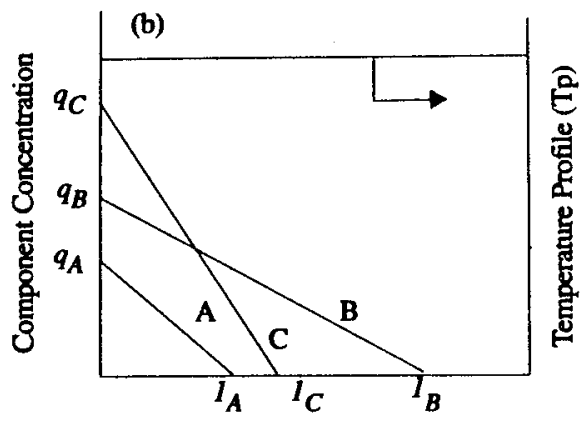

Length of presence in heating zone
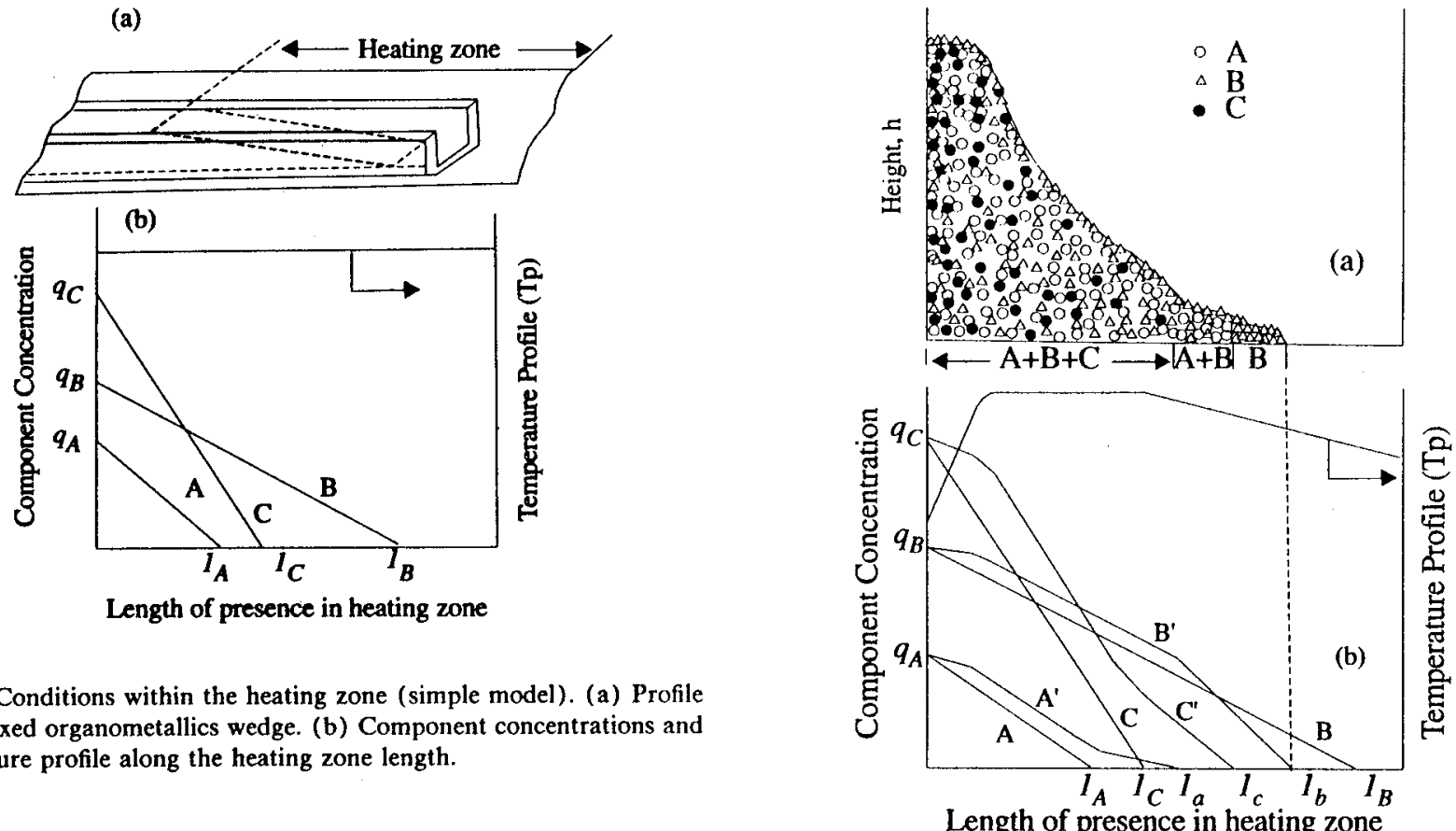

organometallics, moving in the heating zone. The leading edge of the melt wedge, depicted in Fig. 2(a), will consist only of the least volatile component $(B)$ with the quantity of the other two more volatile components, $A$ and $C$, increasing proportionately with the height, $h$, or thickness of the wedge. The equations summarize two important properties of the vaporization process: (a) The mass flow rate of each component vaporized and, therefore, the film deposition rate is simply determined by the cross-sectional area of the organometallics and the velocity with which the carriage is moved through the heating zone, and (b) the ratio of the three compounds in the gas phase, and hence the film's elemental composition, is the same as that in the initial mixture, assuming the film composition is proportional to that of the gas phase. This is a requirement for mass continuity.

If the model is valid, the single source technique should provide a simple means of controlling both the film's composition and growth rate. In practice, however, the preceding assumptions (2) and (3) are not correct. Actually, it is impossible to create a constant temperature plateau, shown in Fig. 2(b), in so narrow a heating zone. Because a very sharp temperature gradient exists, the vaporization rate for each organometallic compound cannot be invariant within the heated zone, because of the strong dependence of volatility on temperature. ${ }^{4}$ The vaporization rate will show an Arrhenius dependency on temperature of the general form

$$
r_{i}=K_{i} \exp \left(-\frac{\Delta H_{i}}{R T}\right),
$$

where $\Delta H_{i}$ is the heat of vaporization for the species $i, R$ the gas constant, and $K_{i}$ is the frequency factor. The composition profiles of the three components, depicted in Fig. 2 (b), are no longer straight lines, but become curves which are related to the temperature profile. Furthermore, the vaporization rates of the organometallic compounds are interdependent.
FIG. 3. Conditions within the heating zone (advanced model). (a) Profile of mixed organometallics wedge, showing the microstructure and nonuniform vaporization surface. (b) Revised component concentrations and temperature profile along heating zone length.

The colligative properties of melting point depression in the mechanical mixture of the organometallics and boiling point elevation in the melt will effect changes in the vaporization rate of individual components. However, the differences in the individual volatilities of the organometallics plays the major role in the interdependence of their rates of vaporization.

From the melt wedge, the most volatile component evaporates fastest and a thicker outer layer becomes the vaporization surface. In this thicker outer layer, the less volatile component becomes more concentrated. A rather complicated microstructure profile develops along the length of the melt within the heated zone, such as the one shown schematically in Fig. 3(a). As the leading edge of the wedge moves through the heating zone, the configuration becomes more and more favorable for the vaporization of the least volatile component because of the increase in the percentage of the melt's total surface area it occupies. Concomitantly, a lowering of the vaporization rates of the more volatile components ensues due to the decrease in the percentage of the melt's surface they occupy. The resulting composition profile along the length of the wedge in the heating zone is shown in Fig. 3(b). A desirable result of this process is that the length $\left(l_{B}\right)$ for complete vaporization of the least volatile component is shortened and the lengths for the more volatile components $\left(l_{A}\right.$ and $\left.l_{C}\right)$ becomes longer. This means the gas phase in the heating zone has a more uniform composition; but, is the gas phase composition still controllable?

As was done earlier, we can derive an expression for the total vaporization rate of each component in the composition profile, shown in Fig. 3(b). The vaporization rate 
of each component $r_{i}$ at position $l$ is given by

$$
r_{i}=w h v \frac{d q_{i}}{w d l}=h v \frac{d q_{i}}{d l}
$$

and the total vaporization rate of each component $R_{i}$ in the heated zone is then

$$
R_{i}=\int r_{i} w d l=\int h v \frac{d q_{i}}{d l} w d l=w h v \int d q_{i}=w h v q_{i},
$$

which is identical to the expression in Eq. (1).

For a typical carrier gas flow rate of $200 \mathrm{sccm}^{3} / \mathrm{min}$ at a pressure of 5 Torr and an effective cross-sectional area of $1 \mathrm{~cm}^{2}$, the linear gas flow velocity is approximately $5 \mathrm{~m} / \mathrm{s}$. Normal values for $l_{i}$ and the carriage velocity $v$ are a few $\mathrm{mm}$ and $0.5 \mathrm{~mm} / \mathrm{min}$, respectively. Therefore, it may be assumed that the vaporized organometallics are swept away immediately to reveal a fresh layer and promote vaporization.

The gas phase concentration of each component, $C_{i}$ is give by

$$
C_{i}=R_{i} \frac{P}{f P_{0}}=w h v \frac{P q_{i}}{f P_{0}},
$$

where $f$ is the total gas flow rate (including oxygen) at the inlet, $P_{0}$ is the atmospheric pressure, and $P$ the system pressure. This simple expression shows the (absolute) gas phase concentration is only determined by the organometallics feed rate, the carrier gas flow rate, and the system's dynamic pressure. This means the film deposition rate and composition can be controlled by the CVD operation parameters, instead of by the volatility of the organometallics, which is exactly what we want.

We have used a single source mixed organometallics vaporizer to produce excellent high- $T_{c}$ superconductive thin films. ${ }^{3}$ The shape and length of the melt wedge in the heating zone is, as expected, a function of the carriage velocity and the wattage of the heating lamp. For the $Y$, $\mathrm{Ba}, \mathrm{Cu} \beta$-diketonates mixture, the leading edge of the melt is believed to be entirely composed of the barium diketonate and the midsection composed of a mixture of yttrium and barium diketonates. This distribution is consistent with the literature ${ }^{5-7}$ in which the $\mathrm{Cu}$ and $\mathrm{Y} \beta$-diketonates are reported to have lower sublimation temperatures than the Ba salt.

In summary, the single source mixed organometallic vaporizer can provide under steady-state conditions a stoichiometric ratio of organometallic vapors which is identical to that in the solid mixture. The film deposition rate is determined only by the carriage velocity and its internal dimensions and the heater wattage. Neither the film composition nor deposition rate are, under normal conditions, influenced by the temperature profile of the heating zone or the volatilities of the organometallics in the mixture.

The model and attendant mathematical derivations presented here are applicable to any multicomponent single source vaporization system, once steady-state conditions are achieved.

The research work was supported by NASA under Contract No. NAG3-1274 and partially supported by the Laboratory for Surface Studies.

\footnotetext{
${ }^{1}$ M. Schieber, S. C. Han, Y. Ariel, S. Chokron, T. Tsach, M. Maharizi, C. Deutscher, D. Racah, A. Raizman, and S. Rotter, J. Cryst. Growth 115, 31 (1991).

${ }^{2}$ R. Hiskes, S. A. Dicarolis, J. L. Yong, S. S. Laderman, R. D. Jacowitz, and R. C. Taber, Appl. Phys. Lett. 59, 606 (1992).

${ }^{3}$ G. Y. Meng, G. Zhou, R. L. Schneider, B. K. Sarma, and M. Levy, Physica C 214 (1993).

${ }^{4}$ Z. H. Yuan, G. Y. Meng, and D. K. Peng, Thin Film Sci. Technol. (in Chinese) 5, 46 (1991).

${ }^{5}$ H. Yu, D. K. Peng, and G. Y. Meng, J. China Univer. Sci. Technol. 16, $66(1986)$.

${ }^{6}$ K. J. Eientraut and R. E. Sievers, J. Am. Chem. Soc. 87, 5254 (1965).

${ }^{7}$ E. W. Berg and N. M. Herrera, Anal. Chem. Acta 60, 117 (1972).
} 